\title{
Convex Hulls of Spheres and Convex Hulls of Convex Polytopes Lying on Parallel Hyperplanes
}

\author{
Menelaos I. Karavelas \\ University of Crete \& FO.R.T.H. \\ Department of Applied Mathematics \\ GR-71409 Heraklion, Crete \\ Greece \\ mkaravel@tem.uoc.gr
}

\author{
Eleni Tzanaki \\ University of Crete \& FO.R.T.H. \\ Department of Applied Mathematics \\ GR-71409 Heraklion, Crete \\ Greece \\ etzanaki@tem.uoc.gr
}

\begin{abstract}
Given a set $\Sigma$ of spheres in $\mathbb{E}^{d}$, with $d \geq 3$ and $d$ odd, having a fixed number of $m$ distinct radii $\rho_{1}, \rho_{2}, \ldots, \rho_{m}$, we show that the worst-case combinatorial complexity of the convex hull $C H_{d}(\Sigma)$ of $\Sigma$ is $\Theta\left(\sum_{1 \leq i \neq j \leq m} n_{i} n_{j}^{\left\lfloor\frac{d}{2}\right\rfloor}\right)$, where $n_{i}$ is the number of spheres in $\Sigma$ with radius $\rho_{i}$. Our bound refines the worst-case upper and lower bounds on the worst-case combinatorial complexity of $C H_{d}(\Sigma)$ for all odd $d \geq 3$.

To prove the lower bound, we construct a set of $\Theta\left(n_{1}+n_{2}\right)$ spheres in $\mathbb{E}^{d}$, with $d \geq 3$ odd, where $n_{i}$ spheres have radius $\rho_{i}, i=1,2$, and $\rho_{2} \neq \rho_{1}$, such that their convex hull has combinatorial complexity $\Omega\left(n_{1} n_{2}^{\left\lfloor\frac{d}{2}\right\rfloor}+n_{2} n_{1}^{\left\lfloor\frac{d}{2}\right\rfloor}\right)$. Our construction is then generalized to the case where the spheres have $m \geq 3$ distinct radii.

For the upper bound, we reduce the sphere convex hull problem to the problem of computing the worst-case combinatorial complexity of the convex hull of a set of $m d$ dimensional convex polytopes lying on $m$ parallel hyperplanes in $\mathbb{E}^{d+1}$, where $d \geq 3$ odd, a problem which is of independent interest. More precisely, we show that the worstcase combinatorial complexity of the convex hull of a set $\left\{\mathcal{P}_{1}, \mathcal{P}_{2}, \ldots, \mathcal{P}_{m}\right\}$ of $m d$-dimensional convex polytopes lying on $m$ parallel hyperplanes of $\mathbb{E}^{d+1}$ is $O\left(\sum_{1<i \neq j<m} n_{i} n_{j}^{\left\lfloor\frac{d}{2}\right\rfloor}\right)$, where $n_{i}$ is the number of vertices of $\mathcal{P}_{i}$. This bound is an improvement over the worst-case bound on the combinatorial complexity of the convex hull of a point set where we impose no restriction on the points' configuration; using the lower bound construction for the sphere convex hull problem, it is also shown to be tight for all odd $d \geq 3$.

Finally: (1) we briefly discuss how to compute convex hulls of spheres with a fixed number of distinct radii, or convex hulls of a fixed number of polytopes lying on parallel hyperplanes; (2) we show how our tight bounds for the parallel polytope convex hull problem, yield tight bounds on the combinatorial complexity of the Minkowski sum of
\end{abstract}

Permission to make digital or hard copies of all or part of this work for personal or classroom use is granted without fee provided that copies are not made or distributed for profit or commercial advantage and that copies bear this notice and the full citation on the first page. To copy otherwise, to republish, to post on servers or to redistribute to lists, requires prior specific permission and/or a fee.

SCG'11, June 13-15, 2011, Paris, France.

Copyright 2011 ACM 978-1-4503-0682-9/11/06 ...\$10.00. two convex polytopes in $\mathbb{E}^{d}$; and (3) we state some open problems and directions for future work.

\section{Categories and Subject Descriptors}

F.2.2 [Analysis of Algorithms and Problem Complexity]: Nonnumerical Algorithms and Problems-Geometrical problems and computations

\section{General Terms}

Algorithms, Theory

\section{Keywords}

high-dimensional geometry, discrete geometry, combinatorial geometry, combinatorial complexity, convex hull, Minkowski sum, spheres, convex polytopes, parallel hyperplanes

\section{INTRODUCTION AND RESULTS}

Let $\Sigma$ be a set of $n$ spheres in $\mathbb{E}^{d}, d \geq 2$, where the dimension $d$ is fixed. We call $\Pi$ a supporting hyperplane of $\Sigma$ if it has non-empty intersection with $\Sigma$ and $\Sigma$ is contained in one of the two closed halfspaces bounded by $\Pi$. We call $H$ a supporting halfspace of the set $\Sigma$ if it contains all spheres in $\Sigma$ and is limited by a supporting hyperplane $\Pi$ of $\Sigma$. The intersection of all supporting halfspaces of $\Sigma$ is called the convex hull $\mathrm{CH}_{d}(\Sigma)$ of $\Sigma$. The definition of convex hulls detailed above is applicable not only to spheres, but also to any finite set of compact geometric objects in $\mathbb{E}^{d}$. In the case of points, i.e., if we have a set $P$ of $n$ points in $\mathbb{E}^{d}$, the worst-case combinatorial complexity ${ }^{1}$ of $C H_{d}(P)$ is known to be $\Theta\left(n^{\left\lfloor\frac{d}{2}\right\rfloor}\right)$. Moreover, there exist worst-case optimal algorithms for constructing $C H_{d}(P)$ that run in $O\left(n^{\left\lfloor\frac{d}{2}\right\rfloor}+n \log n\right)$ time, e.g., see $[15,25,2,24,12]$. Since the complexity of $C H_{d}(P)$ may vary from $O(1)$ to $\Theta\left(n^{\left\lfloor\frac{d}{2}\right\rfloor}\right)$, a lot of work has been devoted to the design of output-sensitive algorithms for constructing $C H_{d}(P)$, i.e., algorithms the running time of which depends on the size of the output convex hull $C H_{d}(P)$, e.g., see [11, $17,27,19,21,13,1,8,9,10]$. For a nice overview of the various algorithms for computing the convex hull of points sets, the interested reader may refer to the paper by Erickson [14], while Avis, Bremner and Seidel [4] have a very nice discussion about the effectiveness of output-sensitive convex hull algorithms for point sets.

\footnotetext{
${ }^{1}$ In the rest of the paper, and unless otherwise stated, we use the term "complexity" to refer to "combinatorial complexity".
} 
Results about the convex hull of non-linear objects are very limited. Aurenhammer [3] showed that the worst-case complexity of the power diagram of a set of $n$ spheres in $\mathbb{E}^{d}, d \geq 2$, is $O\left(n^{\left\lceil\frac{d}{2}\right\rceil}\right)$, which also implies the same upper bound for the worst-case complexity for the convex hull of the sphere set. Rappaport [26] devised an $O(n \log n)$ algorithm for computing the convex hull of a set of discs on the plane, which is worst-case optimal. Boissonnat et al. [6] give an $O\left(n^{\left\lceil\frac{d}{2}\right\rceil}+n \log n\right)$ algorithm for computing the convex hull of a set of $n$ spheres in $\mathbb{E}^{d}, d \geq 2$, which is worst-case optimal in three and even dimensions, since they also show that the worst-case complexity of the convex hull of $n$ spheres in $\mathbb{E}^{3}$ is $\Theta\left(n^{2}\right)$. Finally, their results hold true for the case of homothetic convex objects. Boissonnat and Karavelas [7] settled a conjecture in [6]: they proved that the worst-case complexity of a set of $n$ spheres in $\mathbb{E}^{d}, d \geq 2$, is $\Theta\left(n^{\left\lceil\frac{d}{2}\right\rceil}\right)$, which also implied that the algorithm presented in [6] is optimal for all $d$. As far as output-sensitive algorithms are concerned, Boissonnat, Cérézo and Duquesne [5] showed how to construct the convex hull of a set of $n$ threedimensional spheres in $O(n f)$ time, where $f$ is the size of the output convex hull, while Nielsen and Yvinec [23] discuss optimal or almost optimal output-sensitive convex hull algorithms for planar convex objects.

In this paper we consider the problem of computing the complexity of the convex hull of a set of spheres, when the spheres have a fixed number of distinct radii. This problem has been posed by Boissonnat and Karavelas [7], and it is meaningful for odd dimensions only: in even dimensions the complexity of both the convex hull of $n$ points and the convex hull of $n$ spheres is $\Theta\left(n^{\left\lfloor\frac{d}{2}\right\rfloor}\right)=\Theta\left(n^{\left\lceil\frac{d}{2}\right\rceil}\right)$, i.e., the two bounds match.

Consider a set of $n$ spheres $\Sigma$ in $\mathbb{E}^{d}$, where $d \geq 3$ and $d$ odd, such that the spheres in $\Sigma$ have a fixed number $m$ of distinct radii $\rho_{1}, \rho_{2}, \ldots, \rho_{m}$. Let $n_{i}$ be the number of spheres in $\Sigma$ with radius $\rho_{i}$. We say that $\rho_{\lambda}$ dominates $\Sigma$ if $n_{\lambda}=\Theta(n)$. We further say that $\Sigma$ is uniquely (resp., strongly) dominated, if, for some $\lambda, \rho_{\lambda}$ dominates $\Sigma$, and $n_{i}=o(n)$ (resp., $n_{i}=O(1)$ ), for all $i \neq \lambda$. Using this terminology, we can qualitatively express our results as follows. Firstly, if $\Sigma$ is strongly dominated, then, from the combinatorial complexity point of view, $\mathrm{CH}_{d}(\Sigma)$ behaves as if we had a set of points, or equivalently a set of spheres with the same radius. If, however, $\Sigma$ is dominated by at least two radii, $\mathrm{CH}_{d}(\Sigma)$ behaves as in the generic case, where we impose no restriction on the number of distinct radii in $\Sigma$. Finally, if $\Sigma$ is uniquely dominated (but not strongly dominated), the complexity of $C_{d}(\Sigma)$ stands in-between the two extremes above: the complexity of $\mathrm{CH}_{d}(\Sigma)$ is asymptotically larger than the points' case (or when we have spheres with the same radius), and asymptotically smaller than the generic case, where we impose no restriction on the number of distinct radii in $\Sigma$. From the quantitative point of view, our results refine the results in [7] for any odd dimension $d \geq 3$ as follows. We prove that the worst-case complexity of $C H_{d}(\Sigma)$ is $\Theta\left(\sum_{1 \leq i \neq j \leq m} n_{i} n_{j}^{\left\lfloor\frac{d}{2}\right\rfloor}\right)$. This tight bound constitutes an improvement over the generic worstcase complexity of $\mathrm{CH}_{d}(\Sigma)$ if $\Sigma$ is uniquely dominated, since in this case the complexity of $C H_{d}(\Sigma)$ is $o\left(n^{\left\lceil\frac{d}{2}\right\rceil}\right)$. On the other hard, it matches the generic worst-case complexity of $C H_{d}(\Sigma)$, if $\Sigma$ is dominated by at least two radii: in this case the worst-case complexity of $C H_{d}(\Sigma)$ becomes $\Theta\left(n^{\left\lceil\frac{d}{2}\right\rceil}\right)$. Finally, if $\Sigma$ is strongly dominated, the complexity of $C H_{d}(\Sigma)$ is $\Theta\left(n^{\left\lfloor\frac{d}{2}\right\rfloor}\right)$, i.e., it matches the worst-case complexity of convex hulls of point sets (or sets of spheres where all spheres have the same radius).

To establish the lower bound for the complexity of $C H_{d}(\Sigma)$ we construct a set $\Sigma$ of $\Theta\left(n_{1}+n_{2}\right)$ spheres in $\mathbb{E}^{d}$, for any odd $d \geq 3$, where $n_{1}$ spheres have radius $\rho_{1}$ and $n_{2}$ spheres have radius $\rho_{2} \neq \rho_{1}$, such that worst-case complexity of $C H_{d}(\Sigma)$ is $\Omega\left(n_{1} n_{2}^{\left\lfloor\frac{d}{2}\right\rfloor}+n_{2} n_{1}^{\left\lfloor\frac{d}{2}\right\rfloor}\right)$. This construction is then generalized to sets of spheres having a fixed number of $m \geq 3$ distinct radii. More precisely, we construct a set $\Sigma$ of $n=\sum_{i=1}^{m} n_{i}$ spheres, where $n_{i}$ spheres have radius $\rho_{i}$, with the $\rho_{i}$ 's being pairwise distinct, such that the worst-case complexity of $C H_{d}(\Sigma)$ is $\Omega\left(\sum_{1 \leq i \neq j \leq m} n_{i} n_{j}^{\left\lfloor\frac{d}{2}\right\rfloor}\right)$.

To prove our upper bound we use a lifting map, introduced in [6], that lifts spheres $\sigma_{i}=\left(c_{i}, r_{i}\right)$ in $\mathbb{E}^{d}$ to points $p_{i}=\left(c_{i}, r_{i}\right)$ in $\mathbb{E}^{d+1}$. The convex hull $C H_{d}(\Sigma)$ is then the intersection of the hyperplane $\left\{x_{d+1}=0\right\}$ with the Minkowski sum of the convex hull $\mathrm{CH}_{d+1}(P)$ and the hypercone $\lambda_{0}$, where $P$ is the point set $\left\{p_{1}, p_{2}, \ldots, p_{n}\right\}$ in $\mathbb{E}^{d+1}$, and $\lambda_{0}$ is the lower half hypercone with arbitrary apex, vertical axis and angle at the apex equal to $\frac{\pi}{4}$. When the spheres in $\Sigma$ have a fixed number $m$ of distinct radii, the points of $P$ lie on $m$ hyperplanes parallel to the hyperplane $\left\{x_{d+1}=0\right\}$. In this setting, computing the complexity of $\mathrm{CH}_{d}(\Sigma)$ amounts to computing the complexity of the convex hull of $m$ convex polytopes lying on $m$ parallel hyperplanes of $\mathbb{E}^{d+1}$. This observation gives rise to the second major result in this paper, which is of independent interest, and gives as corollary a tight bound on the worst-case complexity of the Minkowski sum of two convex $d$-polytopes. Given a set $\mathscr{P}=\left\{\mathcal{P}_{1}, \mathcal{P}_{2}, \ldots, \mathcal{P}_{m}\right\}$ of $m$ convex $d$-polytopes in $\mathbb{E}^{d+1}$, with $d \geq 3$ and $d$ odd, we show that the worst-case complexity of the convex hull $C H_{d+1}(\mathscr{P})$ is $\Theta\left(\sum_{1 \leq i \neq j \leq m} n_{i} n_{j}^{\left\lfloor\frac{d}{2}\right\rfloor}\right)$, where $n_{i}$ is the number of vertices of $\mathcal{P}_{i}$. Our upper bound proof is by induction on the number $m$ of parallel hyperplanes. The lower bound follows from the lower bound on the complexity of the convex hull of spheres having $m$ distinct radii. Our bound constitutes an improvement over the worst-case complexity of convex hulls of points sets, if a single polytope of $\mathscr{P}$ has $\Theta(n)$ vertices, whereas all other polytopes have $o(n)$ vertices, where $n$ is the total number of vertices of all $m$ polytopes, while it matches the worstcase complexity of convex hulls of points sets if at least two polytopes have $\Theta(n)$ vertices.

The rest of our paper is structured as follows: In Section 2 we detail our inductive proof of the upper bound on the worst-case complexity of the convex hull of convex polytopes lying on parallel hyperplanes. In Section 3 we prove our upper bound on the worst-case complexity of the convex hull of a set of spheres. Next we present our lower bound construction for any odd $d \geq 3$ in two steps: first for sphere sets with two distinct radii and then for sphere sets with $m \geq 3$ distinct radii. We end the section by discussing how this lower bound yields a tight lower bound for the problem of the previous section. Finally, in Section 4 we summarize our results, we briefly discuss how to compute convex hulls of parallel polytopes and convex hull of spheres, we explain how our results yield tight bounds for the complexity of the Minkowski sum of two convex polytopes, and end with some open problems. 


\section{CONVEX HULLS OF CONVEX POLY- TOPES LYING ON PARALLEL HYPER- PLANES}

A convex polytope, or simply polytope, $\mathcal{P}$ in $\mathbb{E}^{d}$ is the convex hull of a finite set of points $P$ in $\mathbb{E}^{d}$. A polytope $\mathcal{P}$ can equivalently be described as the intersection of all the closed halfspaces containing $P$. A face of $\mathcal{P}$ is an intersection of $\mathcal{P}$ with hyperplanes for which the polytope is contained in one of the two closed halfspaces determined by the hyperplane. The dimension of a face of $\mathcal{P}$ is the dimension of its affine hull. A $k$-face of $\mathcal{P}$ is a $k$-dimensional face of $\mathcal{P}$. We consider the polytope itself as a trivial $d$-dimensional face; all the other faces are called proper faces. We will use the term $d$-polytope to refer to a polytope the trivial face of which is $d$-dimensional. For a $d$-polytope $\mathcal{P}$, the 0 -faces of $\mathcal{P}$ are its vertices, the 1 -faces of $\mathcal{P}$ are its edges, the $(d-2)$-faces of $\mathcal{P}$ are called ridges, while the $(d-1)$-faces are called facets. For $0 \leq k \leq d$ we denote by $f_{k}(\mathcal{P})$ the number of $k$-faces of $\mathcal{P}$. Note that every $k$-face $F$ of $\mathcal{P}$ is also a $k$-polytope whose faces are all the faces of $\mathcal{P}$ contained in $F$. A $k$-simplex in $\mathbb{E}^{d}, k \leq d$, is the convex hull of any $k+1$ affinely independent points in $\mathbb{E}^{d}$. A polytope is called simplicial if all its proper faces are simplices. Equivalently, $\mathcal{P}$ is simplicial if for every vertex $v$ of $\mathcal{P}$ and every face $F \in \mathcal{P}, v$ does not belong to the affine hull of the vertices in $F \backslash\{v\}$.

The $f$-vector $\left(f_{-1}(\mathcal{P}), f_{0}(\mathcal{P}), \ldots, f_{d-1}(\mathcal{P})\right)$ of a simplicial $d$-polytope $\mathcal{P}$ is defined as the $(d+1)$-dimensional vector consisting of the number $f_{k}(\mathcal{P})$ of $k$-faces of $\mathcal{P},-1 \leq k \leq d$, where $f_{-1}(\mathcal{P})=1$ refers to the empty set. The $\bar{h}$-vector $\left(h_{0}(\mathcal{P}), h_{1}(\mathcal{P}), \ldots, h_{d}(\mathcal{P})\right)$ of a simplicial $d$-polytope $\mathcal{P}$ is defined as the $(d+1)$-dimensional vector, where $h_{k}(\mathcal{P}):=$ $\sum_{i=0}^{k}(-1)^{k-i}\left(\begin{array}{c}d-i \\ d-k\end{array}\right) f_{i-1}(\mathcal{P}), 0 \leq k \leq d$. The number $h_{k}(\mathcal{P})$ counts the number of facets of $\mathcal{P}$ in a shelling of $\mathcal{P}$, whose restriction has size $k$; this number is independent of the particular shelling chosen (cf. [28, Theorem 8.19]). It is easy to verify from the defining equations of the $h_{k}(\mathcal{P})$ 's that the elements of the $f$-vector determine the elements of the $h$-vector and vice versa. Moreover, the elements of the $f$-vector (or, equivalently, the $h$-vector) are not linearly independent; they satisfy the so called Dehn-Sommerville equations, which can be written in a very concise form as: $h_{k}(\mathcal{P})=h_{d-k}(\mathcal{P}), 0 \leq k \leq d$. An important implication of the existence of the Dehn-Sommerville equations is that if we know the face numbers $f_{k}(\mathcal{P})$ for all $0 \leq k \leq\left\lfloor\frac{d}{2}\right\rfloor-1$, we can determine the remaining face numbers $f_{k}(\mathcal{P})$ for all $\left\lfloor\frac{d}{2}\right\rfloor \leq k \leq d-1$.

In what follows we recall some facts from [16, Section 5.2] that will be of use to us later. Let $\mathcal{P}$ be a $d$-polytope in $\mathbb{E}^{d}, F$ a facet of $\mathcal{P}$, and $H$ the supporting hyperplane of $F$ (with respect to $\mathcal{P}$ ). For an arbitrary point $p$ in $\mathbb{E}^{d}$, we say that $p$ is beyond (resp., beneath) the facet $F$ of $\mathcal{P}$, if $p$ lies in the open halfspace of $H$ that does not contain $\mathcal{P}$ (resp., contains the interior of $\mathcal{P}$ ). Furthermore, we say that an arbitrary point $v^{\prime}$ is beyond the vertex $v$ of $\mathcal{P}$ if for every facet $F$ of $\mathcal{P}$ containing $v, v^{\prime}$ is beyond $F$, while for every facet $F$ of $\mathcal{P}$ not containing $v, v^{\prime}$ is beneath $F$. The vertices of the polytope $\mathcal{P}^{\prime}=C H_{d}\left((P \backslash\{v\}) \cup\left\{v^{\prime}\right\}\right)$ are the same with those of $\mathcal{P}$, except for $v$ which has been replaced by $v^{\prime}$. In this case we say that $\mathcal{P}^{\prime}$ is obtained from $\mathcal{P}$ by pulling $v$ to $v^{\prime}$. The vertex $v^{\prime}$ of $\mathcal{P}^{\prime}$ does not belong to the affine hull of the vertices in $F^{\prime} \backslash\left\{v^{\prime}\right\}$ for every face $F^{\prime}$ of $\mathcal{P}^{\prime}$. The following result is well-known.
Theorem 1 ([20, 22]). Let $\mathcal{P}$ be a d-polytope.

(i) The d-polytope $\mathcal{P}^{\prime}$ we obtain by pulling a vertex of $\mathcal{P}$ has the same number of vertices with $\mathcal{P}$, and $f_{k}(\mathcal{P}) \leq$ $f_{k}\left(\mathcal{P}^{\prime}\right)$ for all $1 \leq k \leq d-1$.

(ii) The d-polytope $\overline{\mathcal{P}}^{\prime}$ we obtain by successively pulling each of the vertices of $\mathcal{P}$ is simplicial, has the same number of vertices with $\mathcal{P}$, and $f_{k}(\mathcal{P}) \leq f_{k}\left(\mathcal{P}^{\prime}\right)$ for all $1 \leq k \leq d-1$

In the rest of the paper, when we refer to parallel hyperplanes we assume that they have the same unit normal vector, i.e., they have the same orientation. Moreover, if two hyperplanes $\Pi$ and $\Pi^{\prime}$ are parallel, we say that $\Pi^{\prime}$ is above $\Pi$ if $\Pi^{\prime}$ lies in the positive open halfspace delimited by $\Pi$.

Let $\mathscr{P}=\left\{\mathcal{P}_{1}, \mathcal{P}_{2}, \ldots, \mathcal{P}_{m}\right\}$ be a set of $m d$-polytopes lying on $m$ parallel hyperplanes $\Pi_{1}, \Pi_{2}, \ldots, \Pi_{m}$ of $\mathbb{E}^{d+1}$, respectively. Throughout this section we assume that $m \geq 2$ is fixed, and that $\Pi_{j}$ is above $\Pi_{i}$ for all $j>i$. We denote by $P_{i}$ the set of vertices of $\mathcal{P}_{i}$, by $n_{i}$ the cardinality of $P_{i}$, and by $P$ the union $P=P_{1} \cup P_{2} \cup \ldots \cup P_{m}$. Let $\mathcal{P}=C H_{d+1}(P)$; note that, for each $i$, not all vertices in $P_{i}$ are necessarily vertices of $\mathcal{P}$. Furthermore, among the polytopes in $\mathscr{P}$, only $\mathcal{P}_{1}$ and $\mathcal{P}_{m}$ are faces of $\mathcal{P}$.

The theorem that follows is the adaptation of Theorem 1 in the context of $m$ parallel polytopes. Again, we want to perturb the points in $P$ so that $\mathcal{P}^{\prime}$ (the polytope we obtain after perturbing the points in $P$ ) is simplicial with $f_{k}(\mathcal{P}) \leq$ $f_{k}\left(\mathcal{P}^{\prime}\right)$ for $k \geq 1$, but we want to retain the property that the points lying on a hyperplane $\Pi_{i}$, if perturbed, are replaced by points that lie on the same hyperplane. This is almost possible. More precisely, all the faces of the polytope $\mathcal{P}^{\prime}$ are simplicial, with the possible exception of $\mathcal{P}_{1}^{\prime}=\mathcal{P}^{\prime} \cap \Pi_{1}$ and $\mathcal{P}_{m}^{\prime}=\mathcal{P}^{\prime} \cap \Pi_{m}$. Please refer to Section A.1 of the Appendix for the corresponding proof.

Lemma 2. Let $\mathscr{P}=\left\{\mathcal{P}_{1}, \mathcal{P}_{2}, \ldots, \mathcal{P}_{m}\right\}$ be a set of $m \geq 2$ $d$-polytopes lying on $m$ parallel hyperplanes $\Pi_{1}, \Pi_{2}, \ldots, \bar{\Pi}_{m}$ of $\mathbb{E}^{d+1}$, respectively, where $\Pi_{j}$ is above $\Pi_{i}$ for all $j>i$. Let $P_{i}$ be the vertex set of $\mathcal{P}_{i}, 1 \leq i \leq m, P=P_{1} \cup P_{2} \ldots \cup P_{m}$, and $\mathcal{P}=C H_{d+1}(P)$. The points in $P$ can be perturbed in such a way that:

(i) the points of $P$ in each hyperplane $\Pi_{i}$ remain in $\Pi_{i}$, $1 \leq i \leq m$,

(ii) all the faces of $\mathcal{P}^{\prime}$, except possibly the facets $\mathcal{P}_{1}^{\prime}$ and $\mathcal{P}_{m}^{\prime}$, are simplices, and,

(iii) $f_{k}(\mathcal{P}) \leq f_{k}\left(\mathcal{P}^{\prime}\right)$ for all $1 \leq k \leq d$,

where $\mathcal{P}^{\prime}$ is the polytope we obtain after having perturbed the vertices of $\mathcal{P}$ in $P$.

In view of Lemma 2, it suffices to restrict our attention to sets of polytopes $\mathscr{P}$, where $\mathcal{P}$ is simplicial with the possible exception of its two facets $\mathcal{P}_{1}$ and $\mathcal{P}_{m}$. Let $\tilde{\Pi}$ be any hyperplane between and parallel to the hyperplanes $\Pi_{m-1}$ and $\Pi_{m}$ and consider the intersection $\tilde{\mathcal{P}}:=\mathcal{P} \cap \tilde{\Pi}$ (see Fig. 1). Let $\mathcal{F}$ be the set of faces of $\mathcal{P}$ having non-empty intersection with $\tilde{\Pi}$. Note that $\tilde{\mathcal{P}}$ is a $d$-polytope, which is, in general, non-simplicial, and whose proper non-trivial faces are intersections of the form $F \cap \tilde{\Pi}$ where $F \in \mathcal{F}$. Let $A=\left(\alpha_{1}, \alpha_{2}, \ldots, \alpha_{m}\right)$ and $B=\left(\beta_{1}, \beta_{2}, \ldots, \beta_{m}\right)$ be two vectors in $\mathbb{N}^{m}$. We say that $A \preccurlyeq B$ if $\alpha_{i} \leq \beta_{i}$ for all $1 \leq i \leq m$, and denote by $|A|$ the sum of the elements of $\bar{A}$, i.e., $|A|=\sum_{i=1}^{m} \alpha_{i}$. The following lemma provides an upper bound on the number of $k$-faces of $\mathcal{F}$. The proof may be found in Section A.2 of the Appendix. 

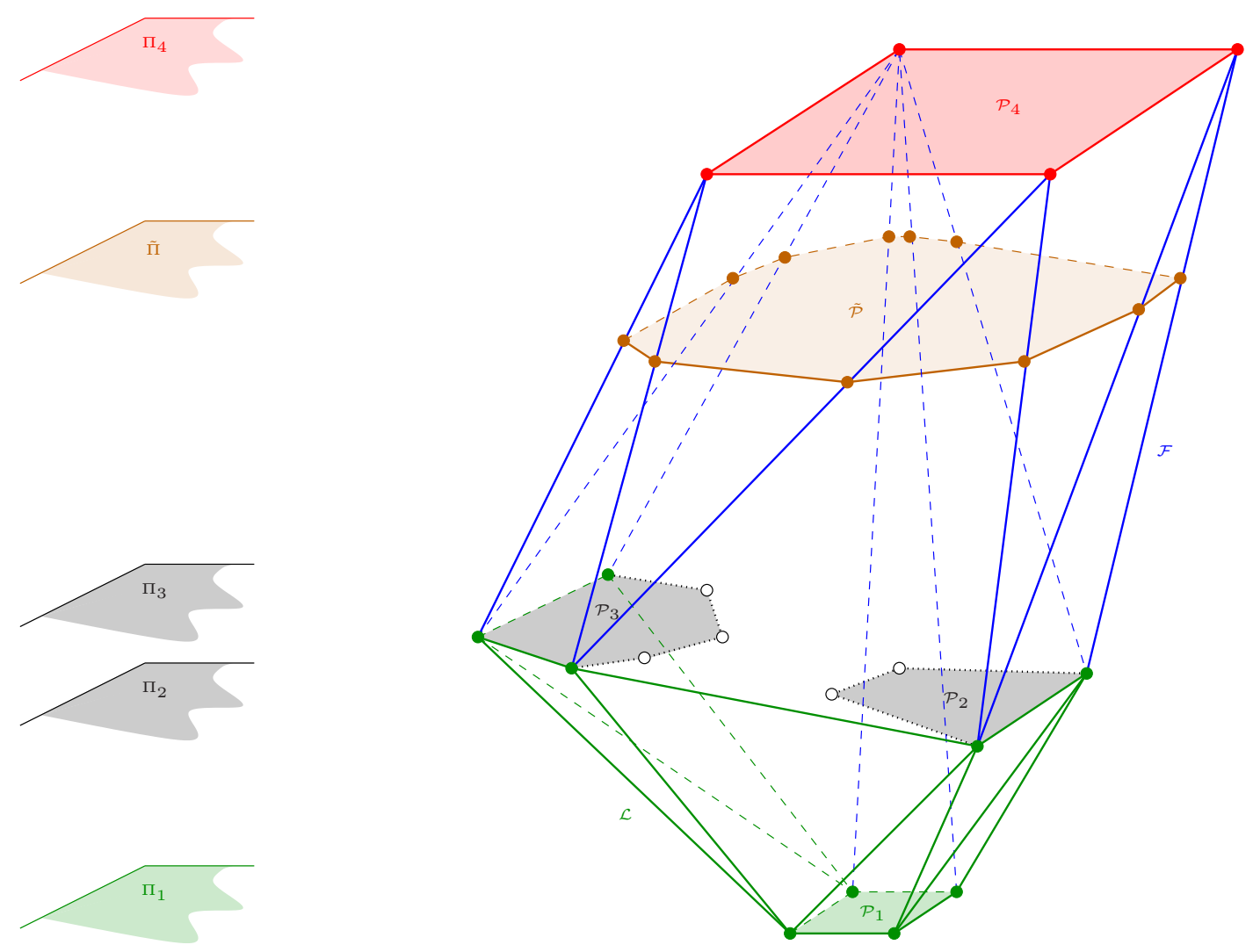

Figure 1: An example of four polytopes lying on four parallel hyperplanes of $\mathbb{E}^{d}$. The white vertices are not vertices of $\mathcal{P}$. The polytopes $\mathcal{P}_{2}$ and $\mathcal{P}_{3}$ are shown in black. All faces of $\mathcal{L}$ are shown in green (only the facet $\mathcal{P}_{1}$ is shown), whereas all faces of $\mathcal{P}_{4}$ are shown in red. The faces in blue are faces of $\mathcal{F}$, whereas the faces in brown are faces of $\tilde{\mathcal{P}}=\mathcal{P} \cap \tilde{\Pi}$.

LEMma 3. The number of $k$-faces of $\mathcal{F}$ is bounded from above as follows:

$$
f_{k}(\mathcal{F}) \leq \sum_{\substack{(0, \ldots, 0,1) \preccurlyeq A \preccurlyeq(k, \ldots, k) \\
|A|=k+1}} \prod_{i=1}^{m}\left(\begin{array}{c}
f_{0}\left(\mathcal{P}_{i}\right) \\
\alpha_{i}
\end{array}\right), \quad 1 \leq k \leq d,
$$

where $\alpha_{i}$ is the $i$-th coordinate of the vector $A \in \mathbb{N}^{m}$.

Exploiting the bounds from Lemma 3 for $1 \leq k \leq\left\lfloor\frac{d}{2}\right\rfloor$, and using the Dehn-Sommerville equations of an appropriately defined simplicial $(d+1)$-polytope containing all faces in $\mathcal{F}$, we derive appropriate asymptotic bounds on $f_{k}(\mathcal{F})$, $1 \leq k \leq d$. Our results are summarized in the following lemma, the proof of which may be found in Section A.3 of the Appendix.

Lemma 4. Let $n_{i}=f_{0}\left(\mathcal{P}_{i}\right), 1 \leq i \leq m$. The following asymptotic bounds hold:

(i) $f_{k}(\mathcal{F})=O\left(n_{m}^{k} \sum_{i=1}^{m-1} n_{i}+n_{m} \sum_{i=1}^{m-1} n_{i}^{k}\right)$, for all $1 \leq$ $k \leq\left\lfloor\frac{d}{2}\right\rfloor$.

(ii) $f_{k}(\mathcal{F})=O\left(n_{m}^{\left\lfloor\frac{d}{2}\right\rfloor} \sum_{i=1}^{m-1} n_{i}+n_{m} \sum_{i=1}^{m-1} n_{i}^{\left\lfloor\frac{d}{2}\right\rfloor}\right)$, for all $\left\lfloor\frac{d+1}{2}\right\rfloor \leq k \leq d$.

We now arrive at the main theorem of this section, concerning the worst-case complexity of the convex hull of a set of $m$ polytopes lying on $m$ parallel hyperplanes.
TheOREM 5. Let $\mathscr{P}=\left\{\mathcal{P}_{1}, \mathcal{P}_{2}, \ldots, \mathcal{P}_{m}\right\}$ be a set of $a$ fixed number of $m \geq 2$ d-polytopes, lying on $m$ parallel hyperplanes of $\mathbb{E}^{d+1}$, where $d \geq 3$ and $d$ is odd. The worst-case complexity of $C H_{d+1}(\mathscr{P})$ is $\Theta\left(\sum_{1 \leq i \neq j \leq m} n_{i} n_{j}^{\left\lfloor\frac{d}{2}\right\rfloor}\right)$, where $n_{i}=f_{0}\left(\mathcal{P}_{i}\right), 1 \leq i \leq m$.

Proof. Let $T(m)$ denote the worst-case complexity of $C H_{d+1}(\mathscr{P})$. We are going to prove, by induction on $m$, that for all $m \geq 1, T(m) \leq c\left(\sum_{1 \leq i \neq j \leq m} n_{i} n_{j}^{\left\lfloor\frac{d}{2}\right\rfloor}+\sum_{i=1}^{m} n_{i}^{\left\lfloor\frac{d}{2}\right\rfloor}\right)$, where $c$ is some appropriately large constant that depends only on $d$.

The case $m=1$ is trivial since the complexity of a $d$ polytope with $n_{1}$ vertices is $O\left(n_{1}^{\left\lfloor\frac{d}{2}\right\rfloor}\right)$. Let us now assume that $m \geq 2$ and that our statement holds for $m-1$; we shall prove it for $m$. To this end, we consider a set of $m$ $d$-polytopes $\mathscr{P}=\left\{\mathcal{P}_{1}, \mathcal{P}_{2}, \ldots, \mathcal{P}_{m}\right\}$, lying on $m$ parallel hyperplanes $\Pi_{1}, \ldots, \Pi_{m}$ of $\mathbb{E}^{d+1}$, such that $\Pi_{j}$ is above $\Pi_{i}$ for all $j>i$. Let $n_{i}=f_{0}\left(\mathcal{P}_{i}\right), 1 \leq i \leq m$, and denote by $\mathcal{P}$ the convex hull $\mathrm{CH}_{d+1}(\mathscr{P})$. Consider a hyperplane $\tilde{\Pi}$ parallel to $\Pi_{m-1}$ and $\Pi_{m}$ and between them, and let $\tilde{\mathcal{P}}=\mathcal{P} \cap \tilde{\Pi}$ (refer to Fig. 1). We denote by $\mathcal{L}$ the set of faces $F$ of $\mathcal{P}$ such that $F \notin \mathcal{P}_{m}$ and $F \cap \tilde{\Pi}=\emptyset$, and by $\mathcal{F}$ the set of faces $F$ of $\mathcal{P}$ with $F \cap \tilde{\Pi} \neq \emptyset$. Clearly, the set of faces of $\mathcal{P}$ is equal to the disjoint union of $\mathcal{L}, \mathcal{F}$ and the set of faces of $\mathcal{P}_{m}$. By the induction hypothesis we have that the number of faces of $C H_{d+1}\left(\mathscr{P} \backslash\left\{\mathcal{P}_{m}\right\}\right)$ is at most $T(m-1)$. Since the 
faces in $\mathcal{L}$ are faces of $C H_{d+1}\left(\mathscr{P} \backslash\left\{\mathcal{P}_{m}\right\}\right)$, we have that the complexity of $\mathcal{L}$ is at most $T(m-1)$. Furthermore, since $\mathcal{P}_{m}$ is a $d$-polytope, its complexity is $O\left(n_{m}^{\left\lfloor\frac{d}{2}\right\rfloor}\right)$. Combining these bounds with the bounds on the number of $k$-faces of $\mathcal{F}$ from Lemma 4, we arrive at the following recurrence relation for $T(m)$ :

$T(m) \leq T(m-1)+O\left(n_{m}^{\left\lfloor\frac{d}{2}\right\rfloor}\right)+O\left(n_{m}^{\left\lfloor\frac{d}{2}\right\rfloor} \sum_{i=1}^{m-1} n_{i}+n_{m} \sum_{i=1}^{m-1} n_{i}^{\left\lfloor\frac{d}{2}\right\rfloor}\right)$.

It is straightforward to verify that $T(m)$ satisfies: $T(m) \leq$ $c\left(\sum_{1 \leq i \neq j \leq m} n_{i} n_{j}^{\left\lfloor\frac{d}{2}\right\rfloor}+\sum_{i=1}^{m} n_{i}^{\left\lfloor\frac{d}{2}\right\rfloor}\right)$, for some appropriately large constant $c$ (that depends only on $d$ ); this establishes the upper bound.

The proof of the lower bound for odd $d \geq 3$ is deferred until Subsection 3.2 (see Corollary 9).

\section{CONVEX HULLS OF SPHERES WITH A FIXED NUMBER OF DISTINCT RADII}

In this section we derive tight upper and lower bounds on the worst-case complexity of the convex hull of a set of spheres in $\mathbb{E}^{d}$ having $m$ distinct radii, where $m$ is considered to be fixed.

Let $\Sigma$ be a set of $n$ spheres $\sigma_{k}=\left(c_{k}, r_{k}\right), 1 \leq k \leq n$, in $\mathbb{E}^{d}$, and let $C H_{d}(\Sigma)$ be the convex hull of the spheres in $\Sigma$. A face of circularity $\ell$ of $C H_{d}(\Sigma), 0 \leq \ell \leq d-1$, is a maximal connected portion of the boundary of $\bar{C} H_{d}(\Sigma)$ consisting of points where the supporting hyperplanes are tangent to a given set of $(d-\ell)$ spheres of $\Sigma$. In the special case where all spheres have the same radius, $C_{d}(\Sigma)$ is combinatorially equivalent to the convex hull $C H_{d}(K)$ of the centers $K$ of spheres in $\Sigma$, in the sense that each face of circularity $\ell$ of $C H_{d}(\Sigma)$ corresponds to a unique $(d-\ell-1)$-face of $C H_{d}(K)$, for $0 \leq \ell \leq d-1$.

We consider here the case where the radii $r_{k}$ can take $m$ distinct values, i.e., $r_{k} \in\left\{\rho_{1}, \rho_{2}, \ldots, \rho_{m}\right\}$. Without loss of generality we may assume that $0<\rho_{1}<\rho_{2}<\ldots<\rho_{m}$. We identify $\mathbb{E}^{d}$ with the hyperplane $H_{0}=\left\{x_{d+1}=0\right\}$ of $\mathbb{E}^{d+1}$ and we call the $(d+1)$-axis of $\mathbb{E}^{d+1}$ the vertical axis, while the expression above will refer to the $(d+1)$-coordinate. Let $\Pi_{i}, 1 \leq i \leq m$, be the hyperplane $\left\{x_{d+1}=\rho_{i}\right\}$ in $\mathbb{E}^{d+1}$, and let $P$ be the point set in $\mathbb{E}^{d+1}$ obtained by mapping each sphere $\sigma_{k}$ to the point $p_{k}=\left(c_{k}, r_{k}\right)$. Let $P_{i}$ denote the subset of $P$ containing points that belong to the hyperplane $\Pi_{i}$, and let $n_{i}$ be the cardinality of $P_{i}$. We denote by $\mathcal{P}$ the convex hull of the points in $P$ (i.e., $\mathcal{P}=C H_{d+1}(P)$ ). We further denote by $\mathcal{P}_{i}$ the convex hull of the points in $P_{i}$ (i.e., $\left.\mathcal{P}_{i}=C H_{d}\left(P_{i}\right)\right)$; more precisely, we identify $\Pi_{i}$ with $\mathbb{E}^{d}$, and then define $\mathcal{P}_{i}$ to be the convex hull of the points in $P_{i}$, seen as points in $\mathbb{E}^{d}$. We use $\mathscr{P}$ to denote the set of the $\mathcal{P}_{i}$ 's. Let $\hat{P}_{i}$ be the subset of $P_{i}$ that defines $\mathcal{P}_{i}$ (i.e., the points in $\hat{P}_{i}$ are the vertices of $\mathcal{P}_{i}$ and thus $\left.\mathcal{P}_{i}=C H_{d}\left(\hat{P}_{i}\right)\right)$, and let $\hat{n}_{i} \leq n_{i}$ be the cardinality of $\hat{P}_{i}$. Finally, let $\hat{P}=\bigcup_{i=1}^{m} \hat{P}_{i}$ and $\hat{\mathcal{P}}=C H_{d+1}(\hat{P})$. Notice that it is possible that $\mathcal{P} \neq \hat{\mathcal{P}}$; such a situation will arise if $P_{1} \neq \hat{P}_{1}$ (resp., $P_{m} \neq \hat{P_{m}}$ ), in which case the intersection of $\mathcal{P}$ with $\Pi_{1}$ (resp., $\Pi_{m}$ ) will consist of more than one $d$-face of $\mathcal{P}$. On the other hand $\mathcal{P}$ and $\hat{\mathcal{P}}$ have the same interior.

Let $\lambda_{0}$ be the half lower hypercone in $\mathbb{E}^{d+1}$ with arbitrary apex, vertical axis, and angle at the apex equal to $\frac{\pi}{4}$. By $\lambda(p)$ we denote the translated copy of $\lambda_{0}$ with apex at $p$; observe that the intersection of the hypercone $\lambda\left(p_{k}\right)$ with the hyperplane $H_{0}$ is identical to the sphere $\sigma_{k}$. Let $\Lambda$ be the set of the lower half hypercones $\left\{\lambda\left(p_{1}\right), \lambda\left(p_{2}\right), \ldots, \lambda\left(p_{n}\right)\right\}$ in $\mathbb{E}^{d+1}$ associated with the spheres of $\Sigma$. The intersection of the convex hull $\mathrm{CH}_{d+1}(\Lambda)$ with $H_{0}$ is equal to $C H_{d}(\Sigma)$.

Let $O^{\prime}$ be a point in the interior of $\mathcal{P}$. We then have the following:

Theorem 6 ([6, TheOrem 1]). Any hyperplane of $\mathbb{E}^{d}$ supporting $\mathrm{CH}_{d}(\Sigma)$ is the intersection with $\mathrm{H}_{0}$ of a unique hyperplane $H$ of $\mathbb{E}^{d+1}$ satisfying the following three properties:

1. $H$ supports $\mathcal{P}$,

2. $H$ is the translated copy of a hyperplane tangent to $\lambda_{0}$ along one of its generatrices,

3. $H$ is above $O^{\prime}$.

Conversely, let $H$ be a hyperplane of $\mathbb{E}^{d+1}$ satisfying the above three properties. Its intersection with $H_{0}$ is a hyperplane of $\mathbb{E}^{d}$ supporting $C H_{d}(\Sigma)$.

Theorem 6 implies an injection $\varphi: C H_{d}(\Sigma) \rightarrow \mathcal{P}$ that maps each face of circularity $(d-\ell-1)$ of $C H_{d}(\Sigma)$ to a unique $\ell$-face of $\mathcal{P}$, for $0 \leq \ell \leq d-1$. Theorem 6 also implies that points in $P_{i} \backslash \hat{P}_{i}, 1 \leq i \leq m$, can never be points on a supporting hyperplane $H$ of $\mathcal{P}$ satisfying the three properties of the theorem. Therefore, $\varphi$ is, in fact, an injection that maps each face of circularity $(d-\ell-1)$ of $C H_{d}(\Sigma)$ to a unique $\ell$-face of $\hat{\mathcal{P}}, 0 \leq \ell \leq d-1$. Observe that $\hat{\mathcal{P}}$ is the convex hull of the set $\mathscr{P}$ of $m$ convex polytopes lying on $m$ parallel hyperplanes of $\mathbb{E}^{d+1}$. By employing Theorem 5 of Section 2, we deduce that $\hat{\mathcal{P}}$ 's complexity is $O\left(\sum_{1 \leq i \neq j \leq m} \hat{n}_{i} \hat{n}_{j}^{\left\lfloor\frac{d}{2}\right\rfloor}\right)=O\left(\sum_{1 \leq i \neq j \leq m} n_{i} n_{j}^{\left\lfloor\frac{d}{2}\right\rfloor}\right)$, which, via the injection $\varphi: C H_{d}(\Sigma) \rightarrow \hat{\mathcal{P}}$, is also an upper bound for the worst-case complexity of $\mathrm{CH}_{d}(\Sigma)$.

\subsection{Lower bound construction with two dis- tinct radii}

For any even dimension $2 \delta$, the trigonometric moment curve $\gamma_{2 \delta}^{t r}(t)$ in $\mathbb{E}^{2 \delta}$ is the curve:

$$
\gamma_{2 \delta}^{t r}(t)=(\cos t, \sin t, \cos 2 t, \sin 2 t, \ldots, \cos \delta t, \sin \delta t),
$$

$t \in[0, \pi)$. Notice that points on $\gamma_{2 \delta}^{t r}(t)$ are points on the sphere of $\mathbb{E}^{2 \delta}$ centered at the origin with radius equal to $\sqrt{\delta}$. For any set $P$ of $n$ points on $\gamma_{2 \delta}^{t r}(t)$, the convex hull $C_{2 \delta}(P)$ is a polytope $\mathcal{Q}$ combinatorially equivalent to the cyclic polytope $C_{2 \delta}(n)$ (cf. $[16,28]$ ). Therefore, $f_{2 \delta-1}(\mathcal{Q})=$ $\Theta\left(n^{\delta}\right)$.

Suppose now that the ambient space is $\mathbb{E}^{d}$, where $d \geq 3$ is odd. Let $H_{1}$ be $H_{2}$ be the hyperplanes $\left\{x_{d}=z_{1}\right\}$ and $\left\{x_{d}=z_{2}\right\}$, where $z_{1}, z_{2} \in \mathbb{R}$ and $z_{2}>z_{1}+2\left(n_{2}+2\right) \sqrt{\delta}$; the quantity $n_{2}$ will be defined below. Consider a set $\Sigma_{1}$ of $n_{1}+1$ points, treated as spheres of $\mathbb{E}^{d}$ of zero radius, on the $(d-1)$-dimensional trigonometric moment curve $\gamma_{d-1}^{\text {tr }}(t)$ embedded in $H_{1}$ (please refer to Fig. 2(left)). Among the $n_{1}+1$ points, the first $n_{1}$ points are chosen with $t \in\left(0, \frac{\pi}{2}\right)$, whereas for the remaining point we require that $t \in\left(\frac{\pi}{2}, \pi\right)$. This implies that the $x_{1}$-coordinate of the first $n_{1}$ points of $\Sigma_{1}$ is positive, whereas the $x_{1}$-coordinate of the last point of $\Sigma_{1}$ is negative. Let $\Sigma_{2}$ be the projection, along the $x_{d}$-axis, of $\Sigma_{1}$ on the hyperplane $H_{2}$. Clearly, the $n_{1}+1$ points of $\Sigma_{2}$ in $H_{2}$ lie on the $(d-1)$-dimensional trigonometric moment curve $\gamma_{d-1}^{\text {tr }}(t)$ embedded in $H_{2}$. The points of $\Sigma_{i}, i=1,2$, lie on a $(d-2)$-dimensional sphere of $\mathbb{E}^{d}$, centered at the point 
$\left(0,0, \ldots, 0, z_{i}\right)$, with radius $\sqrt{\delta}$. Moreover, the number of facets of the polytope $\mathcal{Q}_{i}=C H_{d-1}\left(\Sigma_{i}\right)$ is $\Theta\left(n_{1}^{\left\lfloor\frac{d-1}{2}\right\rfloor}\right)=$ $\Theta\left(n_{1}^{\left\lfloor\frac{d}{2}\right\rfloor}\right)$. The convex hull of the $2\left(n_{1}+1\right)$ points of $\Sigma_{1} \cup \Sigma_{2}$ is a prism $\Delta$. $\Delta$ consists of $\Theta\left(n_{1}^{\left\lfloor\frac{d}{2}\right\rfloor}\right)$ facets not lying on $H_{1}$ or $H_{2}$, called the vertical facets of the prism, the $(d-1)$ face of $C H_{d-1}\left(\Sigma_{1}\right)$, called the bottom facet, and the $(d-1)$ face of $C_{d-1}\left(\Sigma_{2}\right)$, called the top facet. For each vertical facet $F$ of $\Delta$, we denote by $\vec{\nu}_{F}$ the unit normal vector of $F$ pointing outside $\Delta$, and by $F^{+}$(resp., $F^{-}$) the positive (resp., negative) open halfspace delimited by the supporting hyperplane of $F$. Regarding the ridges of $\Delta$, those that are intersections of vertical facets of $\Delta$ will be referred to as vertical ridges. Notice that the vertical ridges of $\Delta$ are perpendicular to $H_{1}$ and $H_{2}$.

Let $Y$ be the oriented hyperplane $\left\{x_{1}=0\right\}$ with unit normal vector $\vec{\nu}=(1,0, \ldots, 0)$. Let also $Y^{+}$and $Y^{-}$be the positive and negative open halfspaces of $\mathbb{E}^{d}$ delimited by $Y$, respectively. $Y$ contains the $x_{d}$-axis, and is perpendicular to the hyperplanes $H_{1}$ and $H_{2}$. Recall that $n_{1}$ points of $\Sigma_{i}$ are contained in $Y^{+}$, whereas exactly one point of $\Sigma_{i}$ is contained in $Y^{-}$. Clearly, $Y$ is in general position with respect to $\Delta, \mathcal{Q}_{1}$ and $\mathcal{Q}_{2}$. Let $\tilde{\mathcal{Q}}_{i}$ be the intersection of $\mathcal{Q}_{i}$ with $Y$, and let $\mathcal{F}_{i}$ be the set of faces of $\mathcal{Q}_{i}$ intersected by $Y . \tilde{\mathcal{Q}}_{i}$ is a $(d-2)$-polytope, and its number of vertices is at most $n_{1}$, since $Y$ cuts at most $n_{1}$ edges of $\mathcal{Q}_{i}$. This implies that the complexity of $\tilde{\mathcal{Q}}_{i}$ is $O\left(n_{1}^{\left\lfloor\frac{d-2}{2}\right\rfloor}\right)=O\left(n_{1}^{\left\lfloor\frac{d}{2}\right\rfloor-1}\right)$; the same bound holds for $\mathcal{F}_{i}$. Since there are no facets of $\Delta$ in $Y^{-}\left(Y^{-}\right.$contains a single vertex of $\left.\mathcal{Q}_{i}\right)$, and since the number of facets of $\mathcal{Q}_{i}$ is $\Theta\left(n_{1}^{\left\lfloor\frac{d}{2}\right\rfloor}\right)$, we conclude that the number of facets of $\mathcal{Q}_{i}$ contained in $Y^{+}$is also $\Theta\left(n_{1}^{\left\lfloor\frac{d}{2}\right\rfloor}\right)$; the same bound holds for the number of vertical facets of $\Delta$ in $Y^{+}$.

Define now a set $\Sigma_{3}=\left\{\sigma_{0}, \sigma_{1}, \ldots, \sigma_{n_{2}+1}\right\}$ of $n_{2}+2$ spheres in $\mathbb{E}^{d}$, where $\sigma_{k}=\left(c_{k}, \rho\right)$, and $c_{k}=(0, \ldots, 0,(2 k+1) \sqrt{\delta})$, $0 \leq k \leq n_{2}+1$. In other words, the sphere $\sigma_{k}$ is centered on the $x_{d}$-axis, with the $d$-th coordinate of its center $c_{k}$ being $(2 k+1) \sqrt{\delta}$, while its radius is $\rho$. We choose $\rho$ to be smaller than $\sqrt{\delta}$, but large enough so that each sphere $\sigma_{i}$ satisfies the following two properties:

(1) it does not intersect any of the ridges of $\Delta$ (including the vertical ridges of $\Delta$ ), and

(2) it intersects the interior of all vertical facets of $\Delta$. Notice also that for this choice for $\rho$, none of the spheres in $\Sigma_{3}$ intersects the hyperplanes $H_{1}$ and $H_{2}$ (recall that $\left.z_{2}>z_{1}+2\left(n_{2}+2\right) \sqrt{\delta}\right)$, while the spheres in $\Sigma_{3}$ are pairwise disjoint; these two observations, however, are not critical for our construction.

We are now going to perturb the centers of the spheres in $\Sigma_{3}$ to get a new set of spheres $\Sigma_{3}^{\prime}$ (see Fig. 2(right)). Define $\sigma_{k}^{\prime}$ to be the sphere with radius $\rho$ and center $c_{k}^{\prime}=$ $c_{k}+\left(\sum_{\ell=0}^{k} \frac{\varepsilon}{2^{\ell}}\right) \vec{\nu}=c_{k}+\varepsilon\left(2-\frac{1}{2^{k}}\right) \vec{\nu}$, where $0<\varepsilon \ll 1$. The quantity $\varepsilon$ is chosen so that the spheres in $\Sigma_{3}^{\prime}$ satisfy almost the same conditions as the spheres in $\Sigma_{3}$. In particular, we require that condition (1) is still satisfied, while we relax the requirement on condition (2): we now require that $\sigma_{k}^{\prime}$ intersects the interior of all vertical facets of $\Delta$ contained in $Y^{+}$. In addition to the two conditions above, we also require that for each $k, 0 \leq k \leq n_{2}+1$, the $(d-2)$-dimensional sphere $\sigma_{k} \cap \sigma_{k}^{\prime}$ is contained in $F^{-}$for all vertical facets $F$ of $\Delta$ in $Y^{+}$.
We will now show that for each pair $\left(\sigma_{k}^{\prime}, F\right)$, where $1 \leq$ $k \leq n_{2}$ and $F$ is a vertical facet of $\Delta$ in $Y^{+}$, the spherical cap $F^{+} \cap \sigma_{k}^{\prime}$ induces a face of circularity $(d-1)$ in $C H_{d}(\Sigma)$. Let $F_{1}$ and $F_{2}$ be the ridges of $\Delta$ on the boundary of $F$ contained in the top and bottom facet, respectively. Finally, let $S_{k}$ be the supporting hyperplane of $\sigma_{k}$ parallel to $F$; we consider $S_{k}$ to be oriented as $F$ (i.e., the unit normal vector of $S_{k}$ is $\vec{\nu}_{F}$ ), and thus $\sigma_{k}$ lies in the closure of the negative halfspace delimited by $S_{k}$. Notice that $S_{k}$ is also a supporting hyperplane for $\Sigma_{3}$. Let $S_{k}^{\prime}$ be the hyperplane we get by translating $S_{k}$ by the vector $\varepsilon\left(2-\frac{1}{2^{k}}\right) \vec{\nu}$. $S_{k}^{\prime}$ supports $\sigma_{k}^{\prime}$, but fails to be a supporting hyperplane for $\Sigma_{3}^{\prime}$. More precisely, $S_{k}^{\prime}$ intersects all spheres $\sigma_{j}^{\prime}$ with $j>k$, whereas all spheres $\sigma_{j}^{\prime}$ with $j<k$, are contained in the negative open halfspace delimited by $S_{k}^{\prime}$. We can, however, perturb $S_{k}^{\prime}$ so that it supports $\Sigma_{3}^{\prime}$ : simply slide $S_{k}^{\prime}$ on sphere $\sigma_{k}^{\prime}$ towards $F_{1}$, while maintaining the property that it remains parallel to $F_{1}$ and $F_{2}$. We keep sliding $S_{k}^{\prime}$ until it has empty intersection with any sphere $\sigma_{j}^{\prime}$ with $j>k$. Notice that due to the way we have perturbed the centers of the spheres in $\Sigma_{3}$ to get $\Sigma_{3}^{\prime}$, the new hyperplane $S_{k}^{\prime \prime}$ we get via this transformation is a supporting hyperplane for $\Sigma_{3}^{\prime}$. In fact, $S_{k}^{\prime \prime}$ is a supporting hyperplane for the sphere set $\Sigma=\Sigma_{1} \cup \Sigma_{2} \cup \Sigma_{3}^{\prime}$ (it touches $C H_{d}(\Sigma)$ at $\sigma_{k}^{\prime}$ only), which implies that $S_{k}^{\prime \prime}$ corresponds to a unique face of circularity $(d-1)$ on $C H_{d}(\Sigma)$.

The same construction can be done for all $k$ with $1 \leq$ $k \leq n_{2}$, and for all vertical facets of $\Delta$ in $Y^{+}$. Since we have $\Theta\left(n_{1}^{\left\lfloor\frac{d}{2}\right\rfloor}\right)$ vertical facets of $\Delta$ in $Y^{+}$, we can construct $n_{2} \Theta\left(n_{1}^{\left\lfloor\frac{d}{2}\right\rfloor}\right)$ distinct supporting hyperplanes of $C H_{d}(\Sigma)$, corresponding to distinct faces of circularity $(d-1)$ on $C H_{d}(\Sigma)$. Hence the complexity of $C H_{d}(\Sigma)$ is $\Omega\left(n_{2} n_{1}^{\left\lfloor\frac{d}{2}\right\rfloor}\right)$. Without loss of generality, we may assume that $n_{2} \leq n_{1}$, in which case we have $n_{2} n_{1}^{\left\lfloor\frac{d}{2}\right\rfloor} \geq \frac{1}{2}\left(n_{2} n_{1}^{\left\lfloor\frac{d}{2}\right\rfloor}+n_{1} n_{2}^{\left\lfloor\frac{d}{2}\right\rfloor}\right)$. Hence, we arrive at the following:

ThEOREM 7. Fix some odd $d \geq 3$. There exists a set $\Sigma$ of spheres in $\mathbb{E}^{d}$, consisting of $n_{i}$ spheres of radius $\rho_{i}, i=1,2$, with $\rho_{1} \neq \rho_{2}$, such that the complexity of the convex hull $C H_{d}(\Sigma)$ is $\Omega\left(n_{1} n_{2}^{\left\lfloor\frac{d}{2}\right\rfloor}+n_{2} n_{1}^{\left\lfloor\frac{d}{2}\right\rfloor}\right)$.

\subsection{Lower bound construction with $m$ distinct radii}

We can easily generalize the lower bound construction of the previous subsection in the case where we have $n_{i}$ spheres of radius $\rho_{i}, 1 \leq i \leq m, m \geq 3$, and the radii $\rho_{i}$ are considered to be mutually distinct.

As in the previous subsection, the ambient space is $\mathbb{E}^{d}$, where $d \geq 3$ is odd. Let $N_{1}=\sum_{i=2}^{m} n_{i}$ and $N_{2}=n_{1}$. We construct the set $\Sigma=\Sigma_{1} \cup \Sigma_{2} \cup \Sigma_{3}^{\prime}$ as in the previous subsection, where $\Sigma_{1}$ and $\Sigma_{2}$ contain each $N_{1}+1$ points, and $\Sigma_{3}^{\prime}$ contains $N_{2}+2$ spheres of some appropriate radius $\rho$ (recall that in the construction of the previous subsection $\rho \approx \sqrt{\frac{d-1}{2}} \geq 1$ ). We then replace $n_{i}$ among the $N_{1}$ points of $\Sigma_{1}$ (resp., $\Sigma_{2}$ ) contained in $Y^{+}$by spheres with the same center and radius equal to $r^{i}$, where $0<r \ll 1$. Furthermore, we replace the unique point of $\Sigma_{1}$ (resp., $\Sigma_{2}$ ) in $Y^{-}$ by a sphere of the same center and radius $r^{2}$. We choose $r$ small enough so that the following two conditions hold:

(1) the prism $\Delta_{r}=C H_{d}\left(\Sigma_{1} \cup \Sigma_{2}\right)$ is combinatorially equivalent $^{2}$ to the prism $\Delta_{0}$ (this is the prism we get for

${ }^{2}$ Combinatorial equivalence here means that each face of 

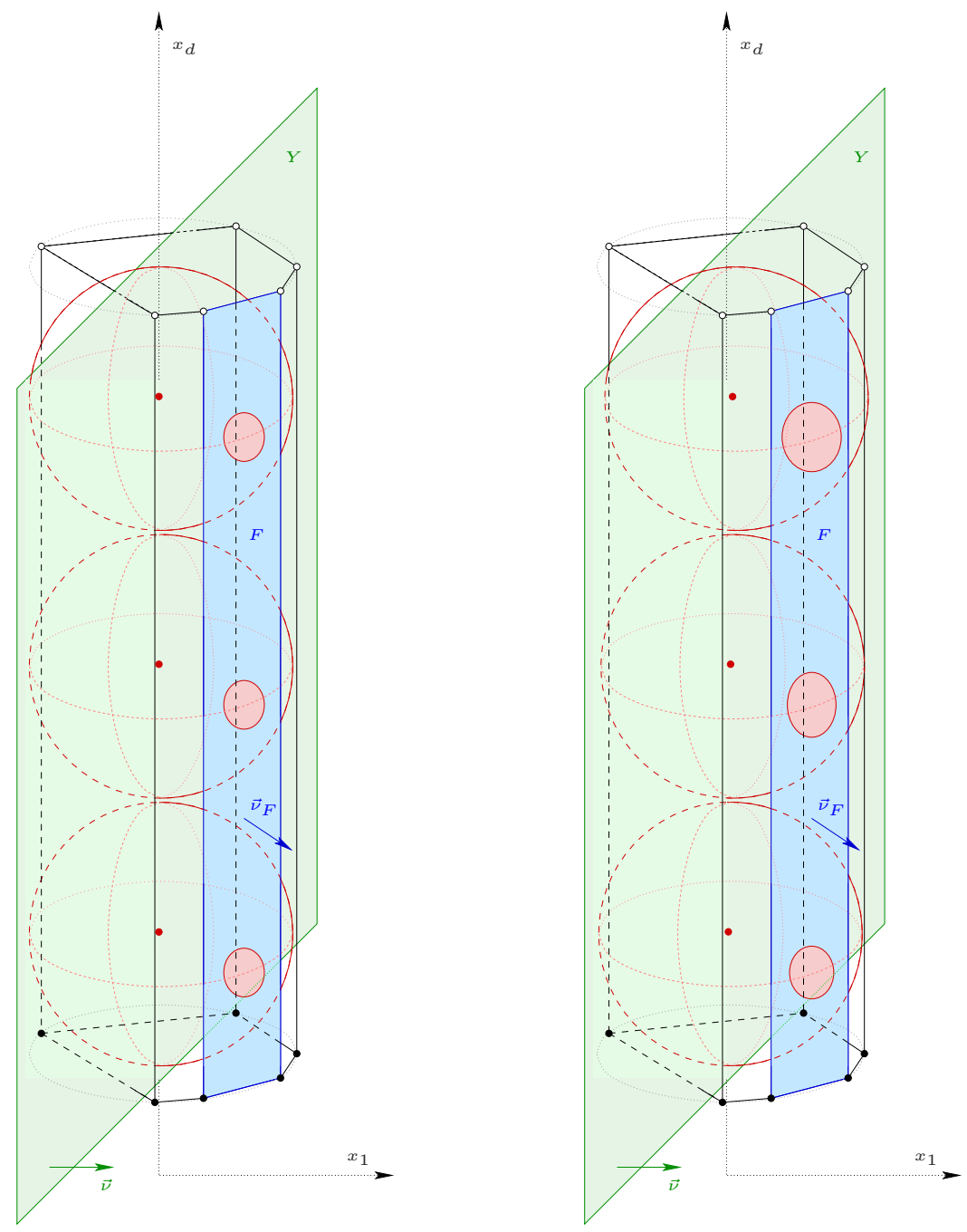

Figure 2: The lower bound construction in the case of two radii. The points in $\Sigma_{1}\left(\right.$ resp., $\left.\Sigma_{2}\right)$ are shown in black (resp., white). The hyperplane $Y$ is shown in green, while the prism $\Delta$ is shown in black. The facet $F$ in blue is one of the vertical facets of $\Delta$ in $Y^{+}$. The sphere sets $\Sigma_{3}$ (left) and $\Sigma_{3}^{\prime}$ (right) are shown in red. The red spherical caps on the left correspond to a unique supporting hyperplane of $C H_{d}\left(\Sigma_{1} \cup \Sigma_{2} \cup \Sigma_{3}\right)$. The red spherical caps on the right correspond to faces of $C H_{d}\left(\Sigma_{1} \cup \Sigma_{2} \cup \Sigma_{3}^{\prime}\right)$ of circularity $(d-1)$.

$r=0$, which is the prism $\Delta$ of the previous subsection), and

(2) the two requirements for the spheres in $\Sigma_{3}^{\prime}$ are still satisfied: each $\sigma_{k}^{\prime}$ does not intersect any of the ridges of $\Delta_{r}$, while each $\sigma_{k}^{\prime}$ intersects the interior of all vertical facets of $\Delta_{r}$ contained in $Y^{+}$.

As described in the previous subsection, the convex hull $C H_{d}(\Sigma)$ of the set $\Sigma=\Sigma_{1} \cup \Sigma_{2} \cup \Sigma_{3}^{\prime}$ of $2\left(N_{1}+1\right)+N_{2}+2$ spheres has $N_{2} \Theta\left(N_{1}^{\left\lfloor\frac{d}{2}\right\rfloor}\right)$ faces of circularity $(d-1)$, and hence its complexity is $\Omega\left(N_{2} N_{1}^{\left\lfloor\frac{d}{2}\right\rfloor}\right)=\Omega\left(n_{1}\left(\sum_{i=2}^{m} n_{i}\right)^{\left\lfloor\frac{d}{2}\right\rfloor}\right)$. Without loss of generality we may assume that $n_{2} \geq n_{1} \geq n_{i}$ for all $3 \leq i \leq m$, in which case we have:

$$
n_{1}\left(\sum_{i=2}^{m} n_{i}\right)^{\left\lfloor\frac{d}{2}\right\rfloor} \geq n_{1} n_{2}^{\left\lfloor\frac{d}{2}\right\rfloor} \geq \frac{1}{m(m-1)}\left(\sum_{1 \leq i \neq j \leq m} n_{i} n_{j}^{\left\lfloor\frac{d}{2}\right\rfloor}\right) .
$$

circularity $\ell$ of $\Delta_{r}$ corresponds to a unique $(d-\ell-1)$-face of $\Delta_{0}$.
Since $m$ is fixed, we conclude that the complexity of $C H_{d}(\Sigma)$ is $\Omega\left(\sum_{1 \leq i \neq j \leq m} n_{i} n_{j}^{\left\lfloor\frac{d}{2}\right\rfloor}\right)$.

THEOREM 8. Fix some odd $d \geq 3$. There exists a set $\Sigma$ of spheres in $\mathbb{E}^{d}$, consisting of $n_{i}$ spheres of radius $\rho_{i}$, $1 \leq i \leq m$, with $\rho_{1}<\rho_{2}<\ldots<\rho_{m}$ and $m \geq 3$ fixed, such that the complexity of $C H_{d}(\Sigma)$ is $\Omega\left(\sum_{1 \leq i \neq j \leq m} n_{i} n_{j}^{\left\lfloor\frac{d}{2}\right\rfloor}\right)$.

Consider again the injection $\varphi: C H_{d}(\Sigma) \rightarrow \hat{\mathcal{P}}$. We have shown above that the worst-case complexity of $C H_{d}(\Sigma)$ is $\Omega\left(\sum_{1<i \neq j<m} n_{i} n_{j}^{\left\lfloor\frac{d}{2}\right\rfloor}\right)$, when $d \geq 3$ is odd and $m \geq 2$ is fixed. Since $\varphi$ is injective, this lower bound also applies to the complexity of $\hat{\mathcal{P}}$. This establishes our lower bound claim in Theorem 5:

Corollary 9. Let $\mathscr{P}=\left\{\mathcal{P}_{1}, \mathcal{P}_{2}, \ldots, \mathcal{P}_{m}\right\}$ be a set of $m$ $d$-polytopes, lying on $m$ parallel hyperplanes of $\mathbb{E}^{d+1}$, with 
$d \geq 3$, $d$ odd, and both $d$ and $m$ are fixed. The worst-case complexity of $C H_{d+1}(\mathscr{P})$ is $\Omega\left(\sum_{1 \leq i \neq j \leq m} n_{i} n_{j}^{\left\lfloor\frac{d}{2}\right\rfloor}\right)$, where $n_{i}=f_{0}\left(\mathcal{P}_{i}\right), 1 \leq i \leq m$.

\section{SUMMARY AND OPEN PROBLEMS}

In this paper we have considered the problem of computing the worst-case complexity of the convex hull $\mathrm{CH}_{d+1}(\mathscr{P})$ of a set $\mathscr{P}=\left\{\mathcal{P}_{1}, \mathcal{P}_{2}, \ldots, \mathcal{P}_{m}\right\}$ of $m$ convex $d$-polytopes lying on $m$ parallel hyperplanes of $\mathbb{E}^{d+1}$, for any odd $d \geq 3$. Denoting by $n_{i}$ the number of vertices of $\mathcal{P}_{i}$, we have shown that the worst-case complexity of the convex hull $\mathrm{CH}_{d+1}(\mathscr{P})$ is $O\left(\sum_{1 \leq j \neq j \leq m} n_{i} n_{j}^{\left\lfloor\frac{d}{2}\right\rfloor}\right)$. This result suggests that, in order to compute $C H_{d+1}(\mathscr{P})$, it pays off to apply an outputsensitive convex hull algorithm to the set of vertices in $\mathscr{P}$. Indeed, for any odd $d \geq 5$ Seidel's shelling algorithm [27], or its modification by Matoušek and Schwarzkopf [21], results in a $O\left(\left(\sum_{1 \leq j \neq j \leq m} n_{i} n_{j}^{\left\lfloor\frac{d}{2}\right\rfloor}\right) \log n\right)$ time algorithm, where $n=$ $\sum_{i=1}^{m} n_{i}$. For $d=3$, the divide-and-conquer algorithm by Chan, Snoeyink and Yap [10] can be competitive against Matoušek and Schwarzkopf's algorithm; hence, we may compute $\mathrm{CH}_{4}(\mathscr{P})$ in $O\left(\min \left\{n^{4 / 3+\epsilon}+\left(\sum_{1 \leq i \neq j \leq m} n_{i} n_{j}\right) \log n\right.\right.$, $\left.\left.\left(\sum_{1<i \neq j<m} n_{i} n_{j}\right) \log ^{2} n\right\}\right)$ time, for any fixed $\epsilon>0$. The above algorithms are nearly optimal for any odd $d \geq 3$; it remains an open problem to compute $\mathrm{CH}_{d+1}(\mathscr{P})$ in worstcase optimal $O\left(\sum_{1 \leq j \neq j \leq m} n_{i} n_{j}^{\left\lfloor\frac{d}{2}\right\rfloor}+n \log n\right)$ time.

A direct consequence of our bound on the complexity of $C H_{d+1}(\mathscr{P})$ is a tight asymptotic bound on the worst-case complexity of the (weighted) Minkowski sum of two polytopes in any odd dimension $d \geq 3$. More precisely, consider a $n$-vertex $d$-polytope $\mathcal{P}$ and a $m$-vertex $d$-polytope $\mathcal{Q}$, and embed them on the hyperplanes $\left\{x_{d+1}=0\right\}$ and $\left\{x_{d+1}=1\right\}$ of $\mathbb{E}^{d+1}$, respectively. The weighted Minkowski sum $(1-\lambda) \mathcal{P} \oplus \lambda \mathcal{Q}, \lambda \in(0,1)$, is combinatorially equivalent to the intersection of $C H_{d+1}(\{\mathcal{P}, \mathcal{Q}\})$ with the hyperplane $\left\{x_{d+1}=\lambda\right\}$, whereas the Minkowski sum $\mathcal{P} \oplus \mathcal{Q}$ is nothing but $\frac{1}{2} \mathcal{P} \oplus \frac{1}{2} \mathcal{Q}$, scaled by a factor of 2 . Applying our results, we deduce that the complexity of $(1-\lambda) \mathcal{P} \oplus \lambda \mathcal{Q}$ (resp., $\mathcal{P} \oplus \mathcal{Q})$ is $\Theta\left(n m^{\left\lfloor\frac{d}{2}\right\rfloor}+m n^{\left\lfloor\frac{d}{2}\right\rfloor}\right)$ for any odd $d \geq 3$. We would like to extend this tight bound to (weighted) Minkowski sums where the number of summands is greater than 2 .

Capitalizing on our result on the complexity of convex hulls of convex polytopes lying on parallel hyperplanes, we have shown that the worst-case complexity of the convex hull $C H_{d}(\Sigma)$ of a set $\Sigma$ of $n$ spheres in $\mathbb{E}^{d}$ with a fixed number of $m$ distinct radii $\rho_{1}, \rho_{2}, \ldots, \rho_{m}$ is $O\left(\sum_{1 \leq j \neq j \leq m} n_{i} n_{j}^{\left\lfloor\frac{d}{2}\right\rfloor}\right)$, for any odd $d \geq 3$, where $n_{i}$ is the number of spheres with radius $\rho_{i}$. By means of an appropriate construction, described in Subsections 3.1 and 3.2, we have shown that the upper bound above is asymptotically tight, implying that our upper bound for $C H_{d+1}(\mathscr{P})$ is also tight. By slightly, but crucially, modifying the algorithm of Boissonnat et al. [6], $C H_{d}(\Sigma)$ may be computed in $O\left(n^{\left\lfloor\frac{d}{2}\right\rfloor}+n \log n+\right.$ $\left.T_{d+1}\left(n_{1}, \ldots, n_{m}\right)\right)$ time, where $T_{d+1}\left(n_{1}, \ldots, n_{m}\right)$ stands for the time needed to compute the convex hull of $m d$-polytopes lying on $m$ parallel hyperplanes in $\mathbb{E}^{d+1}$, where the $i$-th polytope has $n_{i}$ vertices (see [18, Section 5] for details).

Finally, Boissonnat and Karavelas [7] have shown that convex hulls of spheres in $\mathbb{E}^{d}$ and additively weighted Voronoi cells in $\mathbb{E}^{d}$ are combinatorially equivalent. This equivalence suggests that we should be able to refine the worst-case com- plexity of an additively weighted Voronoi cell in any odd dimension, when the number of distinct radii of the spheres involved is considered fixed.

\section{ACKNOWLEDGMENTS}

Partially supported by the FP7-REGPOT-2009-1 project "Archimedes Center for Modeling, Analysis and Computation".

\section{REFERENCES}

[1] Nancy M. Amato and Edgar A. Ramos. On computing Voronoi diagrams by divide-prune-and-conquer. In Proc. 12th Annu. ACM Sympos. Comput. Geom., pages $166-175,1996$.

[2] A. M. Andrew. Another efficient algorithm for convex hulls in two dimensions. Inform. Process. Lett., 9(5):216-219, 1979.

[3] F. Aurenhammer. Power diagrams: properties, algorithms and applications. SIAM J. Comput., 16(1):78-96, 1987.

[4] David Avis, David Bremner, and Raimund Seidel. How good are convex hull algorithms? Comput. Geom. Theory Appl., 7(5-6):265-302, 1997.

[5] J.-D. Boissonnat, A. Cérézo, and J. Duquesne. An output-sensitive algorithm for constructing the convex hull of a set of spheres. In IFIP Conference on Algorithms and efficient computation, September 1992.

[6] Jean-Daniel Boissonnat, André Cérézo, Olivier Devillers, Jacqueline Duquesne, and Mariette Yvinec. An algorithm for constructing the convex hull of a set of spheres in dimension d. Comput. Geom. Theory Appl., 6(2):123-130, 1996.

[7] Jean-Daniel Boissonnat and Menelaos I. Karavelas. On the combinatorial complexity of Euclidean Voronoi cells and convex hulls of $d$-dimensional spheres. In Proc. 14th ACM-SIAM Sympos. Discrete Algorithms (SODA), pages 305-312, 2003.

[8] T. M. Chan. Optimal output-sensitive convex hull algorithms in two and three dimensions. Discrete Comput. Geom., 16(4):361-368, 1996.

[9] T. M. Chan. Output-sensitive results on convex hulls, extreme points, and related problems. Discrete Comput. Geom., 16(4):369-387, 1996.

[10] T. M. Chan, J. Snoeyink, and Chee-Keng Yap. Primal dividing and dual pruning: Output-sensitive construction of four-dimensional polytopes and three-dimensional Voronoi diagrams. Discrete Comput. Geom., 18(4):433-454, 1997.

[11] D. R. Chand and S. S. Kapur. An algorithm for convex polytopes. J. ACM, 17(1):78-86, January 1970.

[12] Bernard Chazelle. An optimal convex hull algorithm in any fixed dimension. Discrete Comput. Geom., 10(1):377-409, 1993.

[13] Bernard Chazelle and J. Matoušek. Derandomizing an output-sensitive convex hull algorithm in three dimensions. Comput. Geom. Theory Appl., 5:27-32, 1995.

[14] Jeff Erickson. New lower bounds for convex hull problems in odd dimensions. SIAM J. Comput., 28(4):1198-1214, 1999. 
[15] L. R. Graham. An efficient algorithm for determining the convex hull of a finite planar set. Inform. Process. Lett., 1(4):132-133, 1972.

[16] Branko Grunbaum. Convex Polytopes, volume 221 of Graduate Texts in Mathematics. Springer-Verlag, New York, 2003.

[17] R. A. Jarvis. On the identification of the convex hull of a finite set of points in the plane. Inform. Process. Lett., 2:18-21, 1973.

[18] Menelaos I. Karavelas and Eleni Tzanaki. Convex hulls of spheres and convex hulls of convex polytopes lying on parallel hyperplanes, March 2011. arXiv:0911.5086v4 [cs.CG] .

[19] D. G. Kirkpatrick and R. Seidel. The ultimate planar convex hull algorithm? SIAM J. Comput., 15:287-299, 1986.

[20] Victor Klee. The number of vertices of a convex polytope. Canadian J. Math., 16:701-720, 1964.

[21] J. Matoušek and O. Schwarzkopf. Linear optimization queries. In Proc. 8th Annu. ACM Sympos. Comput. Geom., pages 16-25, 1992.

[22] P. McMullen. On a problem of Klee concerning convex polytopes. Israel J. Math., 8(1):1-4, 1970.

[23] Frank Nielsen and Mariette Yvinec. Output-sensitive convex hull algorithms of planar convex objects. Internat. J. Comput. Geom. Appl., 8(1):39-66, 1998.

[24] F. Preparata. An optimal real-time algorithm for planar convex hulls. Commun. ACM, 22:402-405, 1979.

[25] F. P. Preparata and S. J. Hong. Convex hulls of finite sets of points in two and three dimensions. Commun. ACM, 20:87-93, 1977.

[26] D. Rappaport. A convex hull algorithm for discs, and applications. Comput. Geom. Theory Appl., 1(3):171-181, 1992.

[27] Raimund Seidel. Constructing higher-dimensional convex hulls at logarithmic cost per face. In Proc. 18th Annu. ACM Sympos. Theory Comput., pages 404-413, 1986.

[28] Günter M. Ziegler. Lectures on Polytopes, volume 152 of Graduate Texts in Mathematics. Springer-Verlag, New York, 1995.

\section{APPENDIX}

\section{A. PROOFS OF LEMMAS 2, 3 AND 4}

\section{A.1 Proof of Lemma 2}

Proof. We construct $\mathcal{P}^{\prime}$ in three steps. Firstly, we properly perturb the points in $\Pi_{1}$ so that $\mathcal{P}_{1}^{\prime}$ is simplicial, then we do the same for the points in $\Pi_{m}$, and, finally, we pull every vertex of $\mathcal{P}$ in $\Pi_{i}$ for $2 \leq i \leq m-1$.

Let $v \in \Pi_{1}$ and choose any $v^{\prime}$ in $\Pi_{1}$ beyond all the facets of $\mathcal{P}$ other than $\mathcal{P}_{1}$ that contain $v$. If we focus our attention on the hyperplane $\Pi_{1}$ and on the polytope $\mathcal{P}_{1} \in \Pi_{1}$ we have that $v$ is a vertex of $\mathcal{P}_{1}$ and $v^{\prime}$ is some point beyond all the facets of $\mathcal{P}_{1}$ containing $v$. We consider the polytope $\mathcal{P}^{\prime}=C H_{d+1}\left((P \backslash\{v\}) \cup\left\{v^{\prime}\right\}\right)$. In view of $[16$, Theorems 5.1.1 $\&$ 5.1.2] the $k$-faces of $\mathcal{P}^{\prime}$ are either:

(i) $k$-faces of $\mathcal{P}_{1}^{\prime}$, or

(ii) $k$-faces of $\mathcal{P}$ not in $\Pi_{1}$ that do not contain $v$, or (iii) faces of the form $C H_{d+1}\left(\left\{v^{\prime}\right\} \cup G_{k-1}\right)$, where $G_{k-1}$ is a $(k-1)$-face not containing $v$ of a facet $F \notin \Pi_{1}$ of $\mathcal{P}$ containing $v$.

The number of $k$-faces of $\mathcal{P}^{\prime}$ does not change due to Case (ii). The number of $k$-faces of $\mathcal{P}^{\prime}$ may increase in Case (iii) if $G_{k-1}$ is contained in a non-simplicial $k$-face of $\mathcal{P}$, otherwise it does not increase. Finally, Case (i) is a consequence of Theorem 1(i).

We set $\mathcal{P}:=\mathcal{P}^{\prime}$ and we repeat the above procedure for every point $v \in P_{1}$. After having perturbed all points in $P_{1}$ we obtain a polytope $\mathcal{P}^{\prime}$ such that $\mathcal{P}_{1}^{\prime}$ is a simplicial polytope. Moreover, we have the additional properties that $f_{k}(\mathcal{P}) \leq f_{k}\left(\mathcal{P}^{\prime}\right)$, for $k \geq 1$, and that for every $v^{\prime} \in \Pi_{1}$ and for each face $F^{\prime} \in \mathcal{P}^{\prime}$ not lying in $\Pi_{1}$, the point $v^{\prime}$ does not lie in the affine hull of the vertices in $F^{\prime} \backslash\left\{v^{\prime}\right\}$. We repeat exactly the same procedure on the points of $\Pi_{m}$. Again, we denote by $\mathcal{P}$ the polytope we obtain after having perturbed the points in $P_{1}$ and $P_{m}$.

It is left to pull the points in the remaining hyperplanes; in fact, we need only perturb the points in $P$ that are vertices of $\mathcal{P}$. Consider a vertex $v$ of $\mathcal{P}$, such that $v \in P_{i}$ for some $2 \leq$ $i \leq m-1$. Let $v^{\prime}$ be any point in $\Pi_{i}$ that lies beyond all the facets of $\mathcal{P}$ containing $v$. The choice of such a $v^{\prime}$ is possible since $\Pi_{i}$ is a hyperplane containing $v$ but not a supporting hyperplane of $\mathcal{P}$. The polytope $\mathcal{P}^{\prime}=C H_{d+1}\left((P \backslash\{v\}) \cup\left\{v^{\prime}\right\}\right)$ is the one we obtain by pulling the vertex $v$ to $v^{\prime}$ and thus from Theorem $1(\mathrm{i})$ we have $f_{k}(\mathcal{P}) \leq f_{k}\left(\mathcal{P}^{\prime}\right)$ for all $k \geq 1$. We continue the same procedure for every vertex $v$ of $\mathcal{P}$ in $\Pi_{i}$, and $2 \leq i \leq m-1$. After having pulled all the vertices of $\mathcal{P}$ in $\Pi_{i}$, for $2 \leq i \leq m-1$, we obtain a polytope $\mathcal{P}^{\prime}$ with the property that for every vertex $v^{\prime}$ of $\mathcal{P}^{\prime}$ in $\Pi_{i}$, with $2 \leq i \leq m-1$, and for every face $F^{\prime} \in \mathcal{P}^{\prime}$, the point $v^{\prime}$ does not lie in the affine hull of the vertices in $F^{\prime} \backslash\left\{v^{\prime}\right\}$.

Summarizing all of the above, we deduce that, after having perturbed all the vertices of $\mathcal{P}$ in $P$, we get a polytope $\mathcal{P}^{\prime}$ with the same number of vertices as $\mathcal{P}$, such that: (1) $f_{k}(\mathcal{P}) \leq f_{k}\left(\mathcal{P}^{\prime}\right)$ for all $k \geq 1,(2) \mathcal{P}_{1}^{\prime}$ and $\mathcal{P}_{m}^{\prime}$ are simplicial, and (3) for every vertex $v^{\prime}$ of $\mathcal{P}^{\prime}$ and every face $F^{\prime} \in \mathcal{P}^{\prime}$, such that $F^{\prime}$ does not lie in $\Pi_{1}$ or $\Pi_{m}$, the vertex $v^{\prime}$ does not lie in the affine hull of the vertices in $F^{\prime} \backslash\left\{v^{\prime}\right\}$. The latter implies that all the faces of $\mathcal{P}^{\prime}$, not in $\Pi_{1}$ or $\Pi_{m}$, are simplices. This completes our proof.

\section{A.2 Proof of Lemma 3}

Proof. According to Lemma 2, it suffices to consider the case where $\mathcal{P}$ is simplicial except possibly for its facets $\mathcal{P}_{1}$ and $\mathcal{P}_{m}$. In this context, a $k$-face $F \in \mathcal{F}$ is simplicial and it is defined by $k+1$ vertices of $P$, where at least one vertex comes from $P_{m}$, whereas the remaining $k$ vertices are vertices of $P_{1}, P_{2}, \ldots, P_{m-1}$. Let $\alpha_{i}$ be the number of vertices of $F$ from $P_{i}$. Clearly, we have $0 \leq \alpha_{i} \leq k$ for $1 \leq i \leq m-1$, $1 \leq \alpha_{m} \leq k$, and $\alpha_{1}+\alpha_{2}+\ldots+\alpha_{m}=k+1$. The maximum number of possible $\left(\alpha_{i}-1\right)$-faces of $\mathcal{P}_{i}$ is $\left(\begin{array}{c}f_{0}\left(\mathcal{P}_{i}\right) \\ \alpha_{i}\end{array}\right)$, which implies that the maximum possible number of $k$-faces of $\mathcal{F}$ is $\prod_{i=1}^{m}\left(\begin{array}{c}f_{0}\left(\mathcal{P}_{i}\right) \\ \alpha_{i}\end{array}\right)$. Summing over all possible values for the $\alpha_{i}$ 's we get the desired expression.

\section{A.3 Proof of Lemma 4}

We start with a few of definitions that will be used in the proof. A polytopal complex $\mathcal{C}$ is a finite collection of polytopes in $\mathbb{E}^{d}$ such that (i) $\emptyset \in \mathcal{C}$, (ii) if $\mathcal{P} \in \mathcal{C}$ then all the faces of $\mathcal{P}$ are also in $\mathcal{C}$ and (iii) the intersection $\mathcal{P} \cap \mathcal{Q}$ for two polytopes in $\mathcal{C}$ is a face of both $\mathcal{P}$ and $\mathcal{Q}$. 
The dimension $\operatorname{dim}(\mathcal{C})$ of $\mathcal{C}$ is the largest dimension of a polytope in $\mathcal{C}$. A polytopal complex is called pure if all its maximal (with respect to inclusion) faces have the same dimension. In this case the maximal faces are called the facets of $\mathcal{C}$. We will use the term $d$-complex to refer to a pure polytopal complex whose facets are $d$-dimensional. A polytopal complex is simplicial if all its faces are simplices. Finally, a polytopal complex $\mathcal{C}^{\prime}$ is called a subcomplex of a polytopal complex $\mathcal{C}$ if all faces of $\mathcal{C}^{\prime}$ are also faces of $\mathcal{C}$.

One important class of polytopal complexes arise from polytopes. More precisely, a $d$-polytope $\mathcal{P}$, together with all its faces and the empty set, form a polytopal $d$-complex, denoted by $\mathcal{C}(\mathcal{P})$. The only maximal face of $\mathcal{C}(\mathcal{P})$, which is clearly the only facet of $\mathcal{C}(\mathcal{P})$, is the polytope $\mathcal{P}$ itself. Moreover, all proper faces of $\mathcal{P}$ form a pure polytopal complex, called the boundary complex $\mathcal{C}(\partial \mathcal{P})$. The facets of $\mathcal{C}(\partial \mathcal{P})$ are just the facets of $\mathcal{P}$, and its $\operatorname{dimension}$ is $\operatorname{dim}(\mathcal{P})-1=d-1$.

Given a polytope $\mathcal{P}$ and a vertex $v$ of $\mathcal{P}$, the star of $v$ is the polytopal complex of all faces of $\mathcal{P}$ that contain $v$, and their faces. The link of $v$ is the subcomplex of the star of $v$ consisting of all the faces of the star of $v$ that do not contain $v$.

We are now ready to state the proof of Lemma 4.

Proof. Since $\left(\begin{array}{c}n_{i} \\ \alpha_{i}\end{array}\right)=\Theta\left(n_{i}^{\alpha_{i}}\right)$ for $\alpha_{i}$ fixed (recall that $\alpha_{i} \leq$ $k \leq d$, and $d$ is fixed), part (i) of the lemma is a direct consequence of Lemma 3 for $1 \leq k \leq\left\lfloor\frac{d}{2}\right\rfloor$ and the fact that $m$ is fixed. The rest of the proof is concerned with part (ii) of the lemma.

Let $\mathcal{K}$ be the polytopal complex whose facets are the $d$ faces $F \in \mathcal{F}$. Clearly, $\mathcal{K}$ is a pure simplicial $d$-complex. Let $\mathcal{L}$ be the set of faces $F$ of $\mathcal{P}$ such that $F \notin \mathcal{P}_{m}$ and $F \cap \tilde{\Pi}=\emptyset$. Finally, let $\partial \mathcal{L}=\mathcal{K} \cap \mathcal{L}$. Let $y$ (resp., $z$ ) be a point below $\Pi_{1}$ (resp., above $\Pi_{m}$ ), such that the vertices of $\mathcal{P}_{1}$ (resp., $\mathcal{P}_{m}$ ) are the only vertices of $\mathcal{P}$ visible from $y$ (resp., $z$ ). Let $Q$ be the set of points consisting of $y, z$, the vertices of $\partial \mathcal{L}$ and the vertices of $\mathcal{P}_{m}$, and let $\mathcal{Q}=C H_{d+1}(Q)$. Observe that the faces of $\mathcal{F}$ are all faces of $\mathcal{Q}$. To see that, first notice that a supporting hyperplane $H_{F}$ for a face $F \in \mathcal{F}$, seen as a face of $\mathcal{P}$, is also a supporting hyperplane for $\mathcal{Q}$, since all vertices in $Q \backslash\{y, z\}$ are also vertices of $\mathcal{P}$, whereas $y$ (resp., $z$ ) is not visible by the vertices of $\mathcal{P}_{m}$ (resp., $\partial \mathcal{L}$ ), and thus $y$ (resp., $z$ ) has to lie in the same halfspace, with respect $H_{F}$, as $\mathcal{P}$. The faces of $\mathcal{Q}$ that are not faces of $\mathcal{F}$ are the faces in the star $\mathcal{S}_{y}$ of $y$ and the star $\mathcal{S}_{z}$ of $z$. To verify this, consider a $k$-face $F$ of $\partial \mathcal{L}$, and let $F_{1}$ be a face in $\mathcal{F}$ that contains $F$. Let $H_{1}$ be a supporting hyperplane of $F_{1}$ with respect to $\mathcal{P}$. Tilt $H_{1}$ until it hits the point $y$, while keeping $H_{1}$ incident to $F_{1}$, and call $H_{1}^{\prime}$ this tilted hyperplane. $H_{1}^{\prime}$ is a supporting hyperplane for $y$ and the vertex set of $\partial \mathcal{L}$, and thus is a supporting hyperplane for $\mathcal{Q}$. The same argument can be applied for the star of $z$. In fact, $\partial \mathcal{L}$ (resp., the boundary complex $\partial \mathcal{P}_{m}$ of $\mathcal{P}_{m}$ ) is nothing but the link of $y$ (resp., $z$ ) in $\mathcal{Q}$.

It is easy to realize that the set of proper $k$-faces of $\mathcal{Q}$ is the disjoint union of the $k$-faces of $\mathcal{F}, \mathcal{S}_{y}$ and $\mathcal{S}_{z}$. This implies that, for $0 \leq k \leq d$ :

$$
f_{k}(\mathcal{Q})=f_{k}(\mathcal{F})+f_{k}\left(\mathcal{S}_{y}\right)+f_{k}\left(\mathcal{S}_{z}\right)
$$

where $f_{0}(\mathcal{F})=0$. The $k$-faces of $\mathcal{Q}$ in $\mathcal{S}_{z}$ are either $k$-faces of $\partial \mathcal{P}_{m}$ or $k$-faces defined by $z$ and a $(k-1)$-face of $\partial \mathcal{P}_{m}$. In fact, there exists a bijection between the $(k-1)$-faces of $\partial \mathcal{P}_{m}$ and the $k$-faces of $\mathcal{S}_{z}$ containing $z$. Hence, we have, for $0 \leq k \leq d$ :

$$
f_{k}\left(\mathcal{S}_{z}\right)=f_{k}\left(\partial \mathcal{P}_{m}\right)+f_{k-1}\left(\partial \mathcal{P}_{m}\right)
$$

where $f_{-1}\left(\partial \mathcal{P}_{m}\right)=1$ and $f_{d}\left(\partial \mathcal{P}_{m}\right)=0$. Analogously, the $k$-faces of $\mathcal{Q}$ in $\mathcal{S}_{y}$ are either $k$-faces of $\partial \mathcal{L}$ or $k$-faces defined by $y$ and a $(k-1)$-face of $\partial \mathcal{L}$. As for $\mathcal{S}_{z}$, there exists a bijection between the $(k-1)$-faces of $\partial \mathcal{L}$ and the $k$-faces of $\mathcal{S}_{y}$ containing $z$. Hence, we have, for $0 \leq k \leq d$ :

$$
f_{k}\left(\mathcal{S}_{y}\right)=f_{k}(\partial \mathcal{L})+f_{k-1}(\partial \mathcal{L})
$$

where $f_{-1}(\partial \mathcal{L})=1$ and $f_{d}(\partial \mathcal{L})=0$.

Let us now turn our attention on deriving bounds for the face numbers $f_{k}(\mathcal{Q})$. Since $\partial \mathcal{P}_{m}$ is the boundary complex of the $n_{m}$-vertex $d$-polytope $\mathcal{P}_{m}$, we have, for $0 \leq k \leq d$ :

$$
f_{k}\left(\partial \mathcal{P}_{m}\right)=O\left(n_{m}^{\left\lfloor\frac{d}{2}\right\rfloor}\right) .
$$

In an analogous manner, since $\partial \mathcal{L}$ is the link of $y$ in $\mathcal{Q}$, it is combinatorially equivalent to a subcomplex of the boundary complex of a $d$-polytope defined over $\sum_{i=1}^{m-1} n_{i}$ vertices. Therefore, for $0 \leq k \leq d$ :

$$
f_{k}(\partial \mathcal{L})=O\left(\left(\sum_{i=1}^{m-1} n_{i}\right)^{\left\lfloor\frac{d}{2}\right\rfloor}\right)=O\left(\sum_{i=1}^{m-1} n_{i}^{\left\lfloor\frac{d}{2}\right\rfloor}\right) .
$$

Combining part (i) of the lemma with relations (1)-(5), we get the following bounds for the lower-dimensional face numbers of $\mathcal{Q}$, i.e., for $0 \leq k \leq\left\lfloor\frac{d}{2}\right\rfloor$ :

$$
f_{k}(\mathcal{Q})=O\left(n_{m}^{\left\lfloor\frac{d}{2}\right\rfloor} \sum_{i=1}^{m-1} n_{i}+n_{m} \sum_{i=1}^{m-1} n_{i}^{\left\lfloor\frac{d}{2}\right\rfloor}\right) .
$$

However, $\mathcal{Q}$ is a simplicial $(d+1)$-polytope, since the facets of $\mathcal{K}$ are simplicial, and since all facets of $\mathcal{S}_{y}$ and $\mathcal{S}_{z}$ are simplicial (the facets of $\mathcal{S}_{y}$ and $\mathcal{S}_{z}$ are defined via simplicial $(d-1)$-faces of $\mathcal{P}$ and the points $y$ and $z$, respectively). Let us recall the defining equations for the elements of the $h$-vector of $\mathcal{Q}$ in terms of the elements of the $f$-vector of $\mathcal{Q}$ :

$$
h_{k}(\mathcal{Q})=\sum_{i=0}^{k}(-1)^{k-i}\left(\begin{array}{l}
d+1-i \\
d+1-k
\end{array}\right) f_{i-1}(\mathcal{Q}),
$$

$0 \leq k \leq d+1$. Combining equations (7) with relations (6), as well as the fact that $f_{-1}(\mathcal{Q})=1$, we get, for $0 \leq k \leq\left\lfloor\frac{d+1}{2}\right\rfloor$ :

$$
h_{k}(\mathcal{Q})=O\left(n_{m}^{\left\lfloor\frac{d}{2}\right\rfloor} \sum_{i=1}^{m-1} n_{i}+n_{m} \sum_{i=1}^{m-1} n_{i}^{\left\lfloor\frac{d}{2}\right\rfloor}\right) .
$$

We are now going to use the Dehn-Sommerville equations for $\mathcal{Q}$ to bound the number of $k$-faces $f_{k}(\mathcal{Q})$ of $\mathcal{Q}$, for $k \geq\left\lfloor\frac{d+1}{2}\right\rfloor$. The Dehn-Sommerville equations can be rewritten as follows (cf. [28, Section 8.4]), for $\left\lfloor\frac{d+1}{2}\right\rfloor \leq k \leq d+1$ :

$f_{k-1}(\mathcal{Q})=\sum_{i=0}^{\frac{d+1}{2}} *\left(\left(\begin{array}{c}d+1-i \\ k-i\end{array}\right)+\left(\begin{array}{c}i \\ k-d-1+i\end{array}\right)\right) h_{i}(\mathcal{Q})$,

where the symbol $\sum_{i=0}^{\frac{\delta}{2}} *$ denotes the sum where the last term is halved if and only if the quantity $\frac{\delta}{2}$ is integral (which is our case since in our setting $\delta=d+1$, which is even). Combining relations (8) and (9), we get, for $\left\lfloor\frac{d+1}{2}\right\rfloor \leq k \leq d+1$ :

$$
f_{k-1}(\mathcal{Q})=O\left(n_{m}^{\left\lfloor\frac{d}{2}\right\rfloor} \sum_{i=1}^{m-1} n_{i}+n_{m} \sum_{i=1}^{m-1} n_{i}^{\left\lfloor\frac{d}{2}\right\rfloor}\right) .
$$

Now using relation $(1)$ with the bounds for $f_{k}\left(\mathcal{S}_{y}\right)$ and $f_{k}\left(\mathcal{S}_{z}\right)$ from relations (2)-(5), we arrive at the following bounds on the number of $k$-faces of $\mathcal{F}$ for $\left\lfloor\frac{d+1}{2}\right\rfloor \leq k \leq d$ :

$$
f_{k}(\mathcal{F})=O\left(n_{m}^{\left\lfloor\frac{d}{2}\right\rfloor} \sum_{i=1}^{m-1} n_{i}+n_{m} \sum_{i=1}^{m-1} n_{i}^{\left\lfloor\frac{d}{2}\right\rfloor}\right) .
$$

This establishes part (ii) of the lemma. 